\title{
The Stochastic Cash Balance Problem with Fixed Costs: The Risk-averse Case
}

\author{
Shuren LIU \\ School of Mathematics and Computational Science, Xiangtan University, Xiangtan 411105, China \\ E-mail:liushuren@xtu.edu.cn \\ Pei TANG \\ School of Mathematics and Computational Science, Xiangtan University, Xiangtan 411105, China \\ E-mail:735389365@qq.com
}

\begin{abstract}
This paper discusses multi-period stochastic cash balance problem with fixed costs when the decision maker is risk averse. By using the consumption model introduced by Chen et al, we characterize the structure of the optimal policy for the stochastic cash balance problem under the general increasing concave utility function and exponential utility function, respectively. We show that the structure of the optimal policy for a decision maker with exponential utility function is almost identical to the structure of the optimal risk-neutral operations policy. Furthermore, we extend the results for the exponential utility function to the ambiguity aversion case.
\end{abstract}

Keywords stochastic cash balance problem; risk aversion; ambiguity aversion; stochastic dynamic programming

\section{Introduction}

The stochastic cash balance problem is an optimization problem faced by a firm, which has to decide how much cash to hold in order to meet its transaction requirement for a given planing horizon with multiple periods. Arrow et al ${ }^{[1]}$ point out that the similarity between the motives of inventories of goods and those for keeping cash balances. In contrast to the usual inventory problem, the stochastic cash balance problem with a case where the cash level (i.e., the checking account level) during the period can either increase or decrease, depending on whether the income is larger or smaller than the expenses during that period. It also allows the decision maker to change the cash level in any direction at the beginning of each period. He can increase the checking account level by withdrawing money from his savings, or decrease it by transferring money to his savings. Therefore, the stochastic cash balance problem can be regarded as a special type of inventory control problems, where the customer demands may be positive or negative and the decision maker can increase or decrease it. Hence, we will use the term inventory level instead of cash level, and also use the terms "order" or "return" to indicate the increase or decrease of the cash levels. At the beginning of each period, the firm may decide to replenish the inventory or return excess stock. Both the ordering cost and the

Received April 1, 2014, accepted September 1, 2014

Supported by National Natural Science Foundation of China (Grant No. 11301445) 
return cost may include a fixed component and a variable component which is proportional to the transaction amount. A holding or penalty cost is charged depending on whether the inventory level is positive or negative. The objective of the firm is to find an ordering or return policy so as to minimize the total expected cost, or equivalently, maximize the total expected profit over the entire planning horizon. Of course, this focus on optimizing expected profit or cost is appropriate for a risk-neutral decision maker, i.e., a firm that is insensitive to profit variations.

The stochastic cash balance problem received considerable amount of attention in the 1960s. Eppen and Fama ${ }^{[2]}$, Whisler ${ }^{[3]}$ consider a cash balance model with independent and identical distribution discrete demands with finite support and without fixed costs. They show the existence of order-up-to and return-down-to levels in the finite and infinite horizon models with discounted cost criterion. Feinberg and Lewis ${ }^{[4]}$ justify the average cost case with the general demand distribution and study the problems with borrowing and lending options and no fixed costs, for which they establish the optimality of simple four-threshold policies. Girgis ${ }^{[5]}$ investigates finite and infinite horizon discounted cost problems with continuous demand when there are fixed costs for increasing or decreasing demand (but not both). Neave ${ }^{[6]}$ studies finite horizon problems with continuous demand when both transactions have fixed costs. However, Chen and Simchi-Levi ${ }^{[7]}$ and Ye and Duenyas ${ }^{[8]}$ notice that some of the claims in $[6]$ are not proved. By using the notion of a $(K, Q)$-convex function introduced by [8], Chen and Simchi$\operatorname{Levi}^{[7]}$ describe the structural properties of optimal solutions of finite horizon cash balance problems when both transactions have fixed costs. Feinberg and Lewis ${ }^{[9]}$ show that structural results stated by [7] indeed hold for finite horizon cash balance problems with discounted criteria and extend the results to the average cost per unit time criteria.

All the papers referenced above assume that the decision makers are risk-neutral. However, many are willing to tradeoff lower expected profit for downside protection against possible losses. Note that traditional stochastic cash balance models fall short of meeting the needs of risk-averse planners. For instance, traditional stochastic cash balance models do not suggest mechanism to reduce the chance of unfavorable profit levels. Thus, it is important to incorporate the notions of risk aversion in the stochastic cash balance problem.

A parallel stream of research studies risk-averse inventory models. Many of the risk-averse inventory models consider single period newsvendor type of models (see, for example, Chen, $\mathrm{Xu}$ and Zhang ${ }^{[10]}$, Eeckhoudt, Gollier and Schlesinger ${ }^{[11]}$, Lau ${ }^{[12]}$, Wu, Zhu and Teunter $\left.{ }^{[13]}\right)$. Bouakiz and Sobel ${ }^{[14]}$ characterize the inventory control strategy so as to minimize the expected utility of the net present value of costs over a finite planing or an infinite horizon. Assuming linear ordering cost, they prove that a base stock policy is optimal. Chen et al ${ }^{[15]}$ propose a general framework to incorporate risk aversion into multi-period inventory models as well as multi-period models that coordinate inventory and pricing strategies. In both cases, they distinguish between models with fixed ordering costs and models with no fixed ordering costs. They show that the structure of the optimal policy for a decision maker with exponential utility function is almost identical to the structure of the optimal risk-neutral inventory (and pricing) policies. These structural results are extended to models in which the decision maker has access to a (partially) complete financial market and can hedge his operational risk through trading 
financial securities.

On the other hand, a decision maker may not know the exact demand distributions and have to estimate them from limited historical data. In this case, the decision maker is ambiguous about the probability distribution. Recently, Nilim and EI Ghaoui ${ }^{[16]}$ study robust solutions to Markov decision problems with uncertain transition matrices. They propose the general idea on the ambiguity averse models, that is, the decision maker choose his policies assuming that nature is adversarial, choosing probability distributions from an ambiguity set to minimize the decision maker's expected utility. Chen and Sun ${ }^{[17]}$ adopt the robust dynamic programming modelling framework introduced by [16] to ambiguity and risk averse inventory and pricing models. They show that the optimal control policies share similar structure properties as Chen et $\mathrm{al}^{[15]}$ for the finite horizon case and extend Chen et $\mathrm{al}^{[15]}$ to including ambiguity aversion and considering infinite horizon models.

In this paper, we propose a framework for incorporating risk aversion in stochastic cash balance problem. We characterize the structure of the optimal policy on the risk-averse stochastic cash balance problem by using the consumption model introduced by Chen et al ${ }^{[15]}$. We show that the structure of the optimal policy for a decision maker with exponential utility function is almost identical to the structure of the optimal risk-neutral operations policy. Furthermore, we extend the results for the exponential utility function to the ambiguity aversion case.

The paper is organized as follows. In Section 2, we propose a model to incorporate risk aversion in the stochastic cash balance problem. In Sections 3 and 4, we focus on characterizing the structure of the optimal policies under the general increasing concave utility function and exponential utility function, respectively. In Section 5 , we extend the results for the exponential utility function to the ambiguity aversion case. Finally, Section 6 is concluding section.

\section{The basic model}

Consider a risk-averse firm facing stochastic demand that has to make ordering or return decisions over a finite planning horizon with a total of $T$ periods.

At the beginning of each period, an ordering or return decision is made. Let $x_{t}$ be the inventory level at the beginning of period $t$ before a decision is made and $y_{t}$ be the inventory level at the beginning of period $t$ after an ordering or return decision is made. Lead time for the ordering or return transaction is assumed to be zero. The transaction cost is denoted by $c\left(x_{t}, y_{t}\right)$, which is calculated as follows:

$$
c\left(x_{t}, y_{t}\right)=\left\{\begin{array}{lll}
K+k\left(y_{t}-x_{t}\right), & \text { if } & y_{t}>x_{t}, \\
0, & \text { if } & y_{t}=x_{t}, \\
Q+q\left(x_{t}-y_{t}\right), & \text { if } & y_{t}<x_{t}
\end{array}\right.
$$

where $K \geq 0, Q \geq 0, k+q \geq 0$. Note that the assumption that $k+q \geq 0$ implies that the unit refund is no more than the unit ordering cost.

For $t=1,2, \cdots, T$, let $p_{t}$ be per unit "sale price" of product in period $t$ and $D_{t}\left(\epsilon_{t}\right)$ (here $\epsilon_{t}$ is a random variable) be "stochastic demand" in period $t$, which consists of obligations paid less funds received (note that the demand in a period can be negative, which corresponds to receiving more funds than were paid out that period). Furthermore, demands in different periods are 
independent of each other. Unsatisfied demand is backlogged. Therefore, the inventory level carried over from period $t$ to the next period, $x_{t+1}$, may be positive or negative. A cost $h_{t}\left(x_{t+1}\right)$ is incurred at the end of period $t$ which represents holding cost when $x_{t+1}>0$ and shortage cost if $x_{t+1}<0$. For technical reasons, we assume that function $h_{t}(x)$ is convex and $\lim _{|x| \rightarrow \infty} h_{t}(x)=\infty$. Further, similar to Assumption 1 in Chen and Simchi-Levi ${ }^{[7]}$, it is assumed that there are finite numbers $x_{t} \leq y_{t} \leq v_{t} \leq z_{t}$ such that $\left(h_{t}\left(y_{t}\right)-h_{t}\left(x_{t}\right)\right) /\left(y_{t}-x_{t}\right)<-k$ and $\left(h_{t}\left(z_{t}\right)-h_{t}\left(v_{t}\right)\right) /\left(z_{t}-v_{t}\right)>q$.

To study the stochastic cash balance problem with fixed costs under risk aversion, we adopt the consumption model under uncertainty introduced by Chen et al ${ }^{[15]}$. The general idea is to directly model consumption, saving and borrowing decisions as well as inventory decisions for the stochastic cash balance problem. Specifically, assume that the decision maker has access to a financial market for borrowing and lending with a risk-free saving and borrowing interest rate $r_{f}$, or equivalently, the discount factor is $\gamma=\frac{1}{1+r_{f}}$. At the beginning of period $t$, assume that the decision maker has initial wealth $w_{t}$ and chooses an operations policy (order or return) that affects his income cash flow. At the end of period $t$, that is, after the uncertainty of this period has been resolved, the decision maker observes his current wealth level $w_{t}+\overline{P_{t}}$ and decides his consumption level $f_{t}$ for the period, where $\overline{P_{t}}$ is the income generated at period $t$. Note that the income at period $t$ is

$\overline{P_{t}}\left(x_{t}, y_{t} ; \epsilon_{t}\right)=-K \delta\left(y_{t}-x_{t}\right)-Q \delta\left(x_{t}-y_{t}\right)-k\left(y_{t}-x_{t}\right)^{+}-q\left(y_{t}-x_{t}\right)^{-}+p_{t} D_{t}\left(\epsilon_{t}\right)-h_{t}\left(y_{t}-D_{t}\left(\epsilon_{t}\right)\right)$,

where $x^{+}=\max \{x, 0\}, x^{-}=\min \{x, 0\}$,

$$
\delta(x)= \begin{cases}1, & \text { if } x>0 \\ 0, & \text { otherwise }\end{cases}
$$

The remaining wealth, $w_{t}+\overline{P_{t}}-f_{t}$, is then saved (or borrowed, if negative) for the next period, i.e., $w_{t+1}=\left(1+r_{f}\right)\left(w_{t}+\overline{P_{t}}-f_{t}\right)$, or, equivalently, $f_{t}=w_{t}-\gamma w_{t+1}+\overline{P_{t}}$. The decision maker's objective is to maximize his expected utility of the consumption flow $E\left[\Pi\left(f_{1}, \cdots, f_{T}\right)\right]$ over the planing horizon $1, \cdots, T$. Moreover, at the last period $T$, we assume the decision maker consumes everything, which corresponds to $w_{T+1}=0$.

According to the consumption model, the decision maker's problem is to find the inventory level $y_{t}$ and decide the initial wealth level $w_{t}$ (or equivalently, the consumption level $f_{t}$ ) for the following optimization problem.

$$
\begin{aligned}
\max & E\left[\Pi\left(f_{1}, f_{2}, \cdots, f_{T}\right)\right] \\
\text { s.t. } & x_{t+1}=y_{t}-D_{t}\left(\epsilon_{t}\right), \\
& f_{t}=w_{t}-\gamma w_{t+1}+\overline{P_{t}}\left(x_{t}, y_{t} ; \epsilon_{t}\right), \\
& w_{T+1}=0 .
\end{aligned}
$$

When the utility function $\Pi\left(f_{1}, f_{2}, \cdots, f_{T}\right)$ takes the linear form $\Pi\left(f_{1}, f_{2}, \cdots, f_{T}\right)=$ $\sum_{t=1}^{T} \gamma^{t-1} f_{t}$, the consumption model reduces to the traditional risk-neutral stochastic cash balance problem. In this case, we denote $V_{t}(x)$ to be the profit-to-go function at the beginning of period $t$ with the initial inventory level $x$. A natural dynamic program for the risk-neutral 
stochastic cash balance problem is as follows:

$$
\begin{aligned}
V_{t}(x)= & \max _{y}\left\{-K \delta(y-x)-Q \delta(x-y)-k(y-x)^{+}-q(y-x)^{-}+p D_{t}\left(\epsilon_{t}\right)-\right. \\
& \left.h_{t}\left(y-D_{t}\left(\epsilon_{t}\right)\right)+\gamma V_{t+1}\left(y-D_{t}\left(\epsilon_{t}\right)\right)\right\} \\
= & \max _{2}\left\{H_{t}(x), \max _{y>x} H_{t}(y)-K-k(y-x), \max _{y<x} H_{t}(y)-Q-q(x-y)\right\},
\end{aligned}
$$

with boundary condition $V_{T+1}(x)=0$, where $H_{t}(x)=E\left\{p D_{t}\left(\epsilon_{t}\right)-h_{t}\left(x-D_{t}\left(\epsilon_{t}\right)\right)+\gamma V_{t+1}(x-\right.$ $\left.\left.D_{t}\left(\epsilon_{t}\right)\right)\right\}$. Without loss of generality, we assume that $K \geq Q$. Define $L_{t} \in \arg \max _{x}\left\{H_{t}(x)-k x\right\}$, $l_{t}=\min \left\{x \mid H_{t}(x)-k x=H_{t}\left(L_{t}\right)-k L_{t}-K\right\}, l_{t}^{\prime}=\min \left\{x \mid H_{t}(x)-k x=H_{t}\left(L_{t}\right)-k L_{t}-(K-Q)\right\}$, $U_{t} \in \arg \max \left\{H_{t}(x)+q x\right\}, u_{t}=\max \left\{x \mid H_{t}(x)+q x=H_{t}\left(U_{t}\right)+q U_{t}-Q\right\}, u_{t}^{\prime}=\min \left\{x \mid H_{t}(x)+\right.$ $\left.q x=H_{t}\left(U_{t}\right)+q U_{t}-(K-Q)\right\}$.

From Lemma 3 in Chen and Simchi-Levi ${ }^{[7]}$, we have $L_{t} \leq U_{t}, l_{t}^{\prime} \leq u_{t}^{\prime}$. Moreover, $u_{t}^{\prime} \leq U_{t}$ due to $K \geq Q \geq 0$. Therefore, the above parameters satisfy the following relationship: $l_{t} \leq$ $l_{t}^{\prime} \leq L_{t} \leq U_{t} \leq u_{t}, l_{t}^{\prime} \leq u_{t}^{\prime} \leq U_{t} \leq u_{t}$.

Notice that these critical points have explicit implications in the stochastic cash balance problem. By definition, $l_{t}$ is the largest value below which one always orders; $l_{t}^{\prime}$ is the smallest value above which one never orders; $u_{t}$ is the smallest value above which one always returns; $u_{t}^{\prime}$ is the largest value below which one never returns. In particular, we call $\left\{l_{t}, l_{t}^{\prime}\right\}$ and $\left\{u_{t}, u_{t}^{\prime}\right\}$ the pairs of order- and return-associated critical points, respectively.

To provide a characterization of the optimal policy, Chen and Simchi-Levi use the following concept of $(K, Q)$-convexity, which is introduced by Ye and Duenyas ${ }^{[8]}$.

Definition 1 A real-valued function is called $(K, Q)$-convex for $K, Q \geq 0$, if for any $x_{0}$, $x_{1}$ with $x_{0} \leq x_{1}$, and $\lambda \in[0,1]$,

$$
f\left((1-\lambda) x_{0}+\lambda x_{1}\right) \leq(1-\lambda) f\left(x_{0}\right)+\lambda f\left(x_{1}\right)+\lambda K+(1-\lambda) Q-\min \{\lambda, 1-\lambda\} \min \{K, Q\} .
$$

A function $f$ is called $(K, Q)$-concave if $-f$ is $(K, Q)$-convex.

See Lemmas 1 and 2 in [7] for the properties of the $(K, Q)$-convex function.

Note that the $(K, 0)$-convexity is exactly the $K$-convexity introduced by $\operatorname{Scarf}^{[18]}$ for the classical stochastic inventory control problem with fixed ordering costs. Moreover, the $(K, K)$ convexity is the symmetric $K$-convexity, a concept introduced and applied in Chen and SimchiLevi ${ }^{[19]}$ to analyze a joint inventory control and pricing problem with fixed ordering costs and a general demand distributions.

Similar to the proof of Theorems 3.1 and 3.2 in [7], we have the following main results for the traditional risk-neutral stochastic cash balance problem.

Theorem 1 Assume that $K \geq Q>0$. The profit-to-go functions $V_{t}(x)$ and $H_{t}(x)$ are $(K, Q)$-concave and the optimal inventory level $y_{t}(x)$ after a decision is made satisfies

$$
y_{t}(x)=\left\{\begin{array}{lll}
L_{t}, & \text { if } & x \leq l_{t}, \\
\in\left\{x, L_{t}\right\}, & \text { if } & x \in\left(l_{t}, l_{t}^{\prime}\right), \\
x, & \text { if } & x \in\left(l_{t}^{\prime}, u_{t}^{\prime}\right), \\
\in\left[l_{t}^{\prime}, x\right], & \text { if } \quad x \in\left(u_{t}^{\prime}, u_{t}\right), \\
U_{t}, & \text { if } \quad x \geq u_{t}
\end{array}\right.
$$


The results for the case $Q \geq K>0$ follow from a symmetric argument.

On a special case of the stochastic cash balance problem where $K=Q>0$, we have

Theorem 2 Assume that $K=Q$. The profit-to-go functions $V_{t}(x)$ and $H_{t}(x)$ are symmetric $K$-concave and the optimal inventory level $y_{t}(x)$ after a decision is made satisfies

$$
y_{t}(x)=\left\{\begin{array}{lll}
L_{t}, & \text { if } \quad x \leq l_{t}, \\
\in\left\{x, L_{t}\right\}, & \text { if } \quad x \in\left(l_{t}, \frac{l_{t}+L_{t}}{2}\right), \\
x, & \text { if } \quad x \in\left[\frac{l_{t}+L_{t}}{2}, \frac{u_{t}+U_{t}}{2}\right], \\
\in\left\{x, U_{t}\right\}, & \text { if } \quad x \in\left(\frac{u_{t}+U_{t}}{2}, u_{t}\right), \\
U_{t}, & \text { if } \quad x \geq u_{t}
\end{array}\right.
$$

\section{Additive increasing concave utility model}

In this section, we focus on the additive general increasing concave utility function. In this case, the objective function of $(1)$ becomes $\Pi\left(f_{1}, \cdots, f_{T}\right)=\sum_{t=1}^{T} \pi_{t}\left(f_{t}\right)$, where the function $\pi_{t}(\cdot)$ is increasing and concave. That is, the utility of the consumption flow is the summation of the utility from the consumption in each period. According to the sequence of events as described before, the optimization model (1) can be solved by the following dynamic programming recursion.

$$
V_{t}(x, w)=\max _{y} E\left[\overline{W_{t}}\left(x, w, y ; \epsilon_{t}\right)\right]
$$

where

$$
\overline{W_{t}}\left(x, w, y ; \epsilon_{t}\right)=\max _{w^{\prime}}\left\{\pi_{t}\left(w-\gamma w^{\prime}+\overline{P_{t}}\left(x, y ; \epsilon_{t}\right)\right)+V_{t+1}\left(y-D_{t}\left(\epsilon_{t}\right), w^{\prime}\right)\right\}
$$

with boundary conditions $V_{T}(x, w)=\pi_{T}\left(w+\overline{P_{T}}\left(x, y ; \epsilon_{T}\right)\right), V_{T+1}(x, 0)=0$. In contrast to risk-neutral stochastic cash problem, here the state variable is two-dimensional, i.e, the current inventory level $x$ and the wealth level $w$.

Instead of working with the dynamic program $(4) \sim(5)$, we find that it is more convenient to work with an equivalent formulation. If $y \geq x$, let $\Pi_{t}^{\prime}(x, w)=V_{t}(x, w-k x)$, and the modified income in period $t$ be $P_{t}^{\prime}\left(y ; \epsilon_{t}\right)=(\gamma k-k) y+(p-\gamma k) D_{t}\left(\epsilon_{t}\right)-h_{t}\left(y_{t}-D_{t}\left(\epsilon_{t}\right)\right)$. In this case, the dynamic program $(4) \sim(5)$ becomes

$$
\Pi_{t}^{\prime}(x, w)=\max _{y \geq x} E\left[W_{t}^{\prime}\left(x, w, y ; \epsilon_{t}\right)\right]
$$

where

$$
W_{t}^{\prime}\left(x, w, y ; \epsilon_{t}\right)=\max _{z^{\prime}}\left\{\pi_{t}\left(w-\gamma z^{\prime}-K \delta(y-x)+P_{t}^{\prime}\left(y ; \epsilon_{t}\right)\right)+\Pi_{t+1}^{\prime}\left(y-D_{t}\left(\epsilon_{t}\right), z^{\prime}\right)\right\}
$$

If $y \leq x$, let $\Pi_{t}^{\prime \prime}(x, w)=V_{t}(x, w+q x)$, and the modified income in period $t$ be $P_{t}^{\prime \prime}\left(y ; \epsilon_{t}\right)=$ $(q-\gamma q) y+(\gamma q-p) D_{t}\left(\epsilon_{t}\right)-h_{t}\left(y_{t}-D_{t}\left(\epsilon_{t}\right)\right)$. In this case, the dynamic program $(4) \sim(5)$ becomes

$$
\Pi_{t}^{\prime \prime}(x, w)=\max _{y \leq x} E\left[W_{t}^{\prime \prime}\left(x, w, y ; \epsilon_{t}\right)\right]
$$

where

$$
W_{t}^{\prime \prime}\left(x, w, y ; \epsilon_{t}\right)=\max _{z^{\prime \prime}}\left\{\pi_{t}\left(w-\gamma z^{\prime \prime}-Q \delta(x-y)+P_{t}^{\prime \prime}\left(y ; \epsilon_{t}\right)\right)+\Pi_{t+1}^{\prime \prime}\left(y-D_{t}\left(\epsilon_{t}\right), z^{\prime \prime}\right)\right\}
$$


Therefore, The dynamic program (4) (5) becomes

$$
\max \left\{\Pi_{t}^{\prime}(x, w), \Pi_{t}^{\prime \prime}(x, w)\right\}
$$

Lemma 1 Assume that $K=0$. In this case, $\Pi_{t}^{\prime}(x, w)$ is jointly concave in $x$ and $w$ for any period $t$. Furthermore, a wealth dependent base stock policy with the base stock level $L_{t}(w)$ is optimal.

Proof We prove the lemma by induction. Obviously, $\Pi_{T+1}^{\prime}(x, w)$ is jointly concave in $x$ and $w$. Assume that $\Pi_{t+1}^{\prime}(x, w)$ is jointly concave in $x$ and $w$. Note that $P_{t}^{\prime}\left(y ; \epsilon_{t}\right)$ is concave in $y$ for any realization of $\epsilon_{t}$. Thus,

$$
W_{t}^{\prime}\left(w, y ; \epsilon_{t}\right)=\max _{z^{\prime}}\left\{\pi_{t}\left(w-\gamma z^{\prime}+P_{t}^{\prime}\left(y ; \epsilon_{t}\right)\right)+\Pi_{t+1}^{\prime}\left(y-D_{t}\left(\epsilon_{t}\right), z^{\prime}\right)\right\}
$$

is jointly concave in $(w, y)$, which further implies that $E\left[W_{t}^{\prime}\left(w, y ; \epsilon_{t}\right)\right]$ is jointly concave in $(w, y)$.

Let $L_{t}(w)$ be an optimal solution for the problem $\max _{y \geq x} E\left[W_{t}^{\prime}\left(w, y ; \epsilon_{t}\right)\right]$. Since $E\left[W_{t}^{\prime}\left(w, y ; \epsilon_{t}\right)\right]$ is concave in $y$ for any fixed $w$, it is optimal to order up to $L_{t}(w)$ when $x<L_{t}(w)$ and not to order otherwise. That is to say, a wealth dependent base stock policy is optimal. Further, according to the properties of the concave function, it is easy to show $\Pi_{t}^{\prime}(x, w)$ is jointly concave in $x$ and $w$. Hence, the lemma follows by induction.

Similar to Lemma 1, we have

Lemma 2 Assume that $Q=0$. In this case, $\Pi_{t}^{\prime \prime}(x, w)$ is jointly concave in $x$ and $w$ for any period $t$. Furthermore, a wealth dependent base return policy with the base return level $U_{t}(w)$ is optimal.

Note that we have $L_{t}(w) \leq U_{t}(w)$. Otherwise, there exists a $x$ such that $U_{t}(w) \leq x \leq L_{t}(w)$. By Lemma 1, it is optimal to order up to $L_{t}(w)$ when $x \leq L_{t}(w)$; By Lemma 2 , it is optimal to reduce down to $U_{t}(w)$ when $x \geq U_{t}(w)$. This is a contradiction.

Due to Lemmas 1 and 2, we have

Theorem 3 Assume that $K=Q=0$, the optimal inventory level $y_{t}^{w}(x)$ after a decision is made satisfies

$$
y_{t}^{w}(x)=\left\{\begin{array}{lll}
L_{t}(w), & \text { if } & x \leq L_{t}(w), \\
x, & \text { if } & x \in\left(L_{t}(w), U_{t}(w)\right), \\
U_{t}(w), & \text { if } & x \geq U_{t}(w)
\end{array}\right.
$$

Recall that in the case of risk-neutral decision maker, Eppen and Fama ${ }^{[2]}$ and Whisler ${ }^{[3]}$ study a special case of the stochastic cash balance problem where $K=Q=0$. They show that in period $t$, there exist two parameters $L_{t}$ and $U_{t}$ with $L_{t} \leq U_{t}$, such that the optimal inventory level $y_{t}(x)$ after a decision is made satisfies

$$
y_{t}(x)=\left\{\begin{array}{lll}
L_{t}, & \text { if } & x \leq L_{t}, \\
x, & \text { if } & x \in\left(L_{t}, U_{t}\right), \\
U_{t}, & \text { if } & x \geq U_{t} .
\end{array}\right.
$$

However, Theorem 3 implies that the optimal policy for the additive increasing concave utility model is different. Indeed, in the risk-averse case, two parameters in the optimal policy depend on the wealth, measured by the position of the risk-free financial security. 


\section{Additive exponential utility function}

In this section, we focus on a special case - the exponential utility function $\pi_{t}(f)=$ $-\alpha_{t} \mathrm{e}^{-\frac{f}{\beta_{t}}}$ with parameters $\alpha_{t}, \beta_{t}>0$, where $\beta_{t}$ is the risk tolerance factor, $\alpha_{t}$ reflects the decision maker's attitude towards the utility obtained from different periods.

According to Chen et al ${ }^{[15]}$, for a risk tolerance parameter $R$, denote the "certainty equivalent" operator with respect to a random variable $\xi$ to be $C E_{\xi}^{R}[\xi]=-R \ln E\left[\mathrm{e}^{-\frac{\xi}{R}}\right]$, which represents the amount of money a decision maker feels indifferent to a gamble with random payoff $\xi$. We also consider the "effective risk tolerance" per period defined as $R_{t}=\sum_{\tau=t}^{T} \gamma^{\tau-t} \beta_{\tau}$. Further, we can obtain the expression $R_{t}\left(1+r_{f}\right)=\left(1+r_{f}\right) \beta_{t}+R_{t+1}$.

The next lemma states that we are able to separately make the operations decisions without considering the wealth/consumption decisions.

Lemma 3 The optimal operations decisions are independent of the wealth/consumption decisions under additive exponential utility function.

Proof We prove the lemma by induction. First, let $P_{t}\left(y_{t} ; \epsilon_{t}\right):=p_{t} D_{t}\left(\epsilon_{t}\right)-h_{t}\left(y_{t}-D_{t}\left(\epsilon_{t}\right)\right)$ in the profit function $\overline{P_{t}}\left(x_{t}, y_{t} ; \epsilon_{t}\right)=-K \delta\left(y_{t}-x_{t}\right)-Q \delta\left(x_{t}-y_{t}\right)-k\left(y_{t}-x_{t}\right)^{+}-q\left(y_{t}-x_{t}\right)^{-}+$ $p_{t} D_{t}\left(\epsilon_{t}\right)-h_{t}\left(y_{t}-D_{t}\left(\epsilon_{t}\right)\right)$ of period $t$.

For $t=T$, we have

$$
\begin{aligned}
& V_{T}(x, w)=\max _{y} E\left[-\alpha_{T} \mathrm{e}^{\frac{-(w-K \delta(y-x)-Q \delta(x-y)-k(y-x)+}{\beta_{T}}}\right] \\
&=\alpha_{T} \mathrm{e}^{\frac{-w}{\beta^{T}}} \max _{y}-\mathrm{e}^{\left.\frac{K \delta(y-x)+Q \delta(x-y)+k(y-x)+}{\beta_{T}}+P_{T}\left(y ; \epsilon_{T}\right)\right)} \\
& E\left[\mathrm{e}^{\frac{-P_{T}\left(y ; \epsilon_{T}\right)}{\beta_{T}}}\right] .
\end{aligned}
$$

Let $G_{T}(x)=\max _{y}\left\{-K \delta(y-x)-Q \delta(x-y)-k(y-x)^{+}-q(y-x)^{-}+C E_{\epsilon_{T}}^{\beta_{T}}\left[P_{T}\left(y ; \epsilon_{T}\right)\right]\right\}$, then $\max _{y}-\mathrm{e}^{\frac{K \delta(y-x)+Q \delta(x-y)+k(y-x)++q(y-x)-}{\beta_{T}}} E\left[\mathrm{e}^{\frac{-P_{T}\left(y ; \epsilon_{t}\right)}{\beta_{T}}}\right]=-\mathrm{e}^{\frac{-G_{T}(x)}{\beta_{T}}}$. Thus, $V_{T}(x, w)=-\alpha_{T} \mathrm{e}^{\frac{-\left(G_{T}(x)+w\right)}{R_{T}}}$.

Suppose that the lemma is true for some $t+1$, i.e., $V_{t+1}(x, w)=-A_{t+1} \mathrm{e}^{\frac{-\left(G_{t+1}(x)+w\right)}{R_{t+1}}}$ for some constant $A_{t+1}>0$. We have

$$
\begin{aligned}
V_{t}(x, w)= & \max _{y} E\left[\operatorname { m a x } _ { w ^ { \prime } } \left\{-\alpha_{t} \mathrm{e}^{\frac{-\left(w-\gamma w^{\prime}-K \delta(y-x)-Q \delta(x-y)-k(y-x)+-q(y-x)-+P_{t}\left(y ; \epsilon_{t}\right)\right)}{\beta_{t}}}\right.\right. \\
& \left.\left.-A_{t+1} \mathrm{e}^{\frac{-\left(G_{t+1}\left(y-D_{t}\left(\epsilon_{t}\right)\right)+w^{\prime}\right)}{R_{t+1}}}\right\}\right] .
\end{aligned}
$$

For any given $y$, the first order optimality condition with respect to $w^{\prime}$ is

$$
\frac{1}{\beta_{t}} \alpha_{t} \mathrm{e}^{\frac{-\left(w-\gamma w^{\prime}\right)}{\beta_{t}}} \mathrm{e}^{\frac{K \delta(y-x)+Q \delta(x-y)+k(y-x)++q(y-x)--P_{t}\left(y ; \epsilon_{t}\right)}{\beta_{t}}}=\frac{1}{\gamma R_{t+1}} A_{t+1} \mathrm{e}^{\frac{-w^{\prime}}{R_{t+1}}} \mathrm{e}^{\frac{-G_{t+1}\left(y-D_{t}\left(\epsilon_{t}\right)\right)}{R_{t+1}}}
$$

equivalently,

$$
\begin{aligned}
\ln \frac{\alpha_{t}}{\beta_{t}}-\frac{w-\gamma w^{\prime}}{\beta_{t}}+ & \frac{K \delta(y-x)+Q \delta(x-y)+k(y-x)^{+}+q(y-x)^{-}-P_{t}\left(y ; \epsilon_{t}\right)}{\beta_{t}} \\
& =\ln \frac{A_{t+1}}{\gamma R_{t+1}}-\frac{w^{\prime}}{R_{t+1}}-\frac{G_{t+1}\left(y-D_{t}\left(\epsilon_{t}\right)\right)}{R_{t+1}} .
\end{aligned}
$$

Thus, at state $(x, w)$, for any given $y$ and the realization of the current period uncertainty $\epsilon_{t}$, the optimal banking decision $w_{*}^{\prime}$ is

$$
w_{*}^{\prime}=-\frac{\beta_{t}}{R_{t}} G_{t+1}\left(y-D_{t}\left(\epsilon_{t}\right)\right)+\frac{R_{t+1}}{R_{t}}\left(-K \delta(y-x)-Q \delta(x-y)-k(y-x)^{+}-q(y-x)^{-}\right.
$$




$$
\left.+P_{t}\left(y ; \epsilon_{t}\right)\right)+\frac{R_{t+1}}{R_{t}} w+\frac{R_{t+1} \beta_{t}}{R_{t}} \ln \frac{A_{t+1} \beta_{t}}{\gamma \alpha_{t} R_{t+1}},
$$

which implies that the optimal consumption decision in period $t$ is

$$
\begin{aligned}
f_{t}^{\prime}= & \frac{\beta_{t}}{R_{t}}\left[w+\left(-K \delta(y-x)-Q \delta(x-y)-k(y-x)^{+}-q(y-x)^{-}+P_{t}\left(y ; \epsilon_{t}\right)\right)+\right. \\
& \left.\gamma G_{t+1}\left(y-D_{t}\left(\epsilon_{t}\right)\right)\right]-\frac{\gamma R_{t+1} \beta_{t}}{R_{t}} \ln \frac{A_{t+1} \beta_{t}}{\gamma \alpha_{t} R_{t+1}} .
\end{aligned}
$$

Furthermore, by Eq (12), we have

$$
\begin{aligned}
V_{t}(x, w) & =\frac{R_{t}}{\gamma R_{t+1}} A_{t+1} \max _{y} E\left[-\mathrm{e}^{\frac{\left(w_{*}^{\prime}+G_{t+1}\left(y-D_{t}\left(\epsilon_{t}\right)\right)\right.}{R_{t+1}}}\right] \\
& =A_{t} \mathrm{e}^{-\frac{w}{R_{t}}} \max _{y} E\left[-\mathrm{e}^{-\frac{\left.\gamma G_{t+1}\left(y-D_{t}\left(\epsilon_{t}\right)\right)-K \delta(y-x)-Q \delta(x-y)-k(y-x)^{+}-q(y-x)-+P_{t}\left(y ; \epsilon_{t}\right)\right)}{R_{t}}}\right],
\end{aligned}
$$

where $A_{t}=\frac{R_{t}}{\gamma R_{t+1}} A_{t+1}\left(\frac{A_{t+1} \beta_{t}}{\gamma \alpha_{t} R_{t+1}}\right)^{-\frac{\beta_{t}}{R_{t}}}>0$. Let

$$
\begin{aligned}
G_{t}(x)= & \max _{y}\left\{-K \delta(y-x)-Q \delta(x-y)-k(y-x)^{+}-q(y-x)^{-}\right. \\
& \left.-R_{t} \ln E\left[\mathrm{e}^{\left\{-\frac{1}{R_{t}}\left[P_{t}\left(y ; \epsilon_{t}\right)+\gamma G_{t+1}\left(y-D_{t}\left(\epsilon_{t}\right)\right)\right]\right\}}\right]\right\} \\
= & \max _{y}\left\{-K \delta(y-x)-Q \delta(x-y)-k(y-x)^{+}-q(y-x)^{-}\right. \\
& \left.+C E_{\epsilon_{t}}^{R_{t}}\left[P_{t}\left(y ; \epsilon_{t}\right)+\gamma G_{t+1}\left(y-D_{t}\left(\epsilon_{t}\right)\right)\right]\right\}
\end{aligned}
$$

then $V_{t}(x, w)=-A_{t} \mathrm{e}^{\frac{-\left(G_{t}(x)+w\right)}{R_{t}}}$. Hence, the lemma follows by induction.

Therefore, by Lemma 3, the stochastic cash balance problem under additive exponential utility function reduces to the optimal problem (13) with boundary condition $G_{T+1}(x)=0$.

To present our main result for the problem with $K>0$ and $Q>0$, we need the following proposition.

Proposition 1 If a function $f(x, \xi)$ is $(K, Q)$-concave in $x$ for any realization of $\xi$, then for any $R>0$ the function

$$
g(x)=C E_{\xi}^{R}[f(x, \xi)]
$$

is also $(K, Q)$-concave.

Proof Let $M(x)=E\left[\exp (f(x, \xi)]\right.$. For any $x_{0}, x_{1}$ with $x_{0} \leq x_{1}$ and $\lambda \in[0,1], x_{\lambda}=$ $(1-\lambda) x_{0}+\lambda x_{1}$, We have

$$
\begin{aligned}
M\left(x_{\lambda}\right) \leq & E\left[\exp \left((1-\lambda) f\left(x_{0}, \xi\right)+\lambda f\left(x_{1}, \xi\right)+\lambda K+(1-\lambda) Q-\min \{\lambda, 1-\lambda\} \min \{K, Q\}\right)\right] \\
= & \exp (\lambda K) \exp ((1-\lambda) Q) \exp (-\min \{\lambda, 1-\lambda\} \min \{K, Q\}) \\
& E\left[\exp \left((1-\lambda) f\left(x_{0}, \xi\right)\right) \exp \left(\lambda f\left(x_{1}, \xi\right)\right)\right] \\
\leq & \exp (\lambda K) \exp ((1-\lambda) Q) \exp (-\min \{\lambda, 1-\lambda\} \min \{K, Q\}) \\
& E\left[\exp \left(f\left(x_{0}, \xi\right)\right]^{1-\lambda} E\left[\exp \left(f\left(x_{1}, \xi\right)\right)\right]^{\lambda}\right. \\
= & M\left(x_{0}\right)^{1-\lambda} M\left(x_{1}\right)^{\lambda} \exp (\lambda K) \exp ((1-\lambda) Q) \exp (-\min \{\lambda, 1-\lambda\} \min \{K, Q\}),
\end{aligned}
$$

where the first inequality holds since $f(\cdot)$ is $(K, Q)$-convex and the second inequality follows from the Hölder inequality with $\frac{1}{p}=1-\lambda$ and $\frac{1}{q}=\lambda$.

Note that Proposition 1 also holds for $K$-concave and symmetric- $K$-concave function since $K$-concave and symmetric- $K$-concave are both special cases of $(K, Q)$-concave function. 
We can now present the optimal policy for the risk-averse stochastic case balance problem with additive exponential utility function. Without loss of generality, we assume that $K \geq Q \geq$ 0 .

Let

$$
H_{t}^{\mathrm{e}}(x)=C E_{\epsilon_{t}}^{R_{t}}\left[P_{t}\left(x ; \epsilon_{t}\right)+\gamma G_{t+1}\left(x-D_{t}\left(\epsilon_{t}\right)\right)\right] .
$$

Define $L_{t}^{\mathrm{e}} \in \arg \max _{x}\left\{H_{t}^{\mathrm{e}}(x)-k x\right\}, l_{t}^{\mathrm{e}}=\min \left\{x \mid H_{t}^{\mathrm{e}}(x)-k x=H_{t}^{\mathrm{e}}\left(L_{t}^{\mathrm{e}}\right)-k L_{t}^{\mathrm{e}}-K\right\}, l_{t}^{\mathrm{e}}=$ $\min \left\{x \mid H_{t}^{\mathrm{e}}(x)-k x=H_{t}^{\mathrm{e}}\left(L_{t}^{\mathrm{e}}\right)-k L_{t}^{\mathrm{e}}-(K-Q)\right\}, U_{t}^{\mathrm{e}} \in \arg \max \left\{H_{t}^{\mathrm{e}}(x)+q x\right\}, u_{t}^{\mathrm{e}}=\max \left\{x \mid H_{t}^{\mathrm{e}}(x)+\right.$ $\left.q x=H_{t}^{\mathrm{e}}\left(U_{t}^{\mathrm{e}}\right)+q U_{t}^{\mathrm{e}}-Q\right\}, u_{t}^{\prime \mathrm{e}}=\min \left\{x \mid H_{t}^{\mathrm{e}}(x)+q x=H_{t}^{\mathrm{e}}\left(U_{t}^{\mathrm{e}}\right)+q U_{t}^{\mathrm{e}}-(K-Q)\right\}$.

Then, with Proposition 1, similar to Theorems 1 and 2, we have the following main results for the additive exponential utility model with $K>0$ and $Q>0$.

Theorem 4 Assume that $K \geq Q>0 . G_{t}(x)$ and $H_{t}^{\mathrm{e}}(x)$ are $(K, Q)$-concave and the optimal inventory level $y_{t}^{\mathrm{e}}(x)$ after a decision is made satisfies

$$
y_{t}^{\mathrm{e}}(x)=\left\{\begin{array}{lll}
L_{t}^{\mathrm{e}}, & \text { if } & x \leq l_{t}^{\mathrm{e}}, \\
\in\left\{x, L_{t}^{\mathrm{e}}\right\}, & \text { if } & x \in\left(l_{t}^{\mathrm{e}}, l_{t}^{\prime}\right), \\
x, & \text { if } & x \in\left(l_{t}^{\prime}, u_{t}^{\prime}\right), \\
\in\left[l_{t}^{\prime}, x\right], & \text { if } & x \in\left(u_{t}^{\prime}{ }^{\mathrm{e}}, u_{t}^{\mathrm{e}}\right), \\
U_{t}^{\mathrm{e}}, & \text { if } \quad x \geq u_{t}^{\mathrm{e}}
\end{array}\right.
$$

The results for the case $Q \geq K>0$ follow from a symmetric argument.

On a special case of the stochastic cash balance problem where $K=Q>0$, we have

Theorem 5 Assume that $K=Q . G_{t}(x)$ and $H_{t}^{\mathrm{e}}(x)$ are symmetric $K$-concave and the optimal inventory level $y_{t}^{\mathrm{e}}(x)$ after a decision is made satisfies

$$
y_{t}^{\mathrm{e}}(x)=\left\{\begin{array}{lll}
L_{t}^{\mathrm{e}}, & \text { if } & x \leq l_{t}^{\mathrm{e}}, \\
\in\left\{x, L_{t}^{\mathrm{e}}\right\}, & \text { if } & x \in\left(l_{t}^{\mathrm{e}}, \frac{l_{t}^{\mathrm{e}}+L_{t}^{\mathrm{e}}}{2}\right), \\
x, & \text { if } & x \in\left[\frac{l_{t}^{\mathrm{e}}+L_{t}^{\mathrm{e}}}{2}, \frac{u_{t}^{\mathrm{e}}+U_{t}^{\mathrm{e}}}{2}\right], \\
\in\left\{x, U_{t}^{\mathrm{e}}\right\}, & \text { if } & x \in\left(\frac{u_{t}^{\mathrm{e}}+U_{t}^{\mathrm{e}}}{2}, u_{t}^{\mathrm{e}}\right), \\
U_{t}^{\mathrm{e}}, & \text { if } & x \geq u_{t}^{\mathrm{e}}
\end{array}\right.
$$

\section{Additive exponential utility function with ambiguity aversion}

In this section, we introduce the finite horizon ambiguity averse model under exponential utility function. Specially, assume that the decision maker does not know the exact probability distribution for the random variable $\epsilon_{t}$. Rather, the decision maker is only aware of a set of probability distributions to which the probability distribution of $\epsilon_{t}$ belongs. According to Chen and Sun $^{[17]}$, in period $t$, the decision maker choose his policies assuming that nature is adversarial, choosing probability distributions $g_{\epsilon_{t}}$ from an ambiguity set $\Omega_{t}$ to minimize the decision maker's expected utility. Thus, similar to (4) (5), a dynamic program for the risk and ambiguity averse stochastic cash balance problem is as follows:

$$
V_{t}(x, w)=\max _{y} \min _{g_{\epsilon_{t}} \in \Omega_{t}} E_{g_{\epsilon_{t}}}\left[\overline{W_{t}}\left(x, w, y ; \epsilon_{t}\right)\right]
$$


where

$$
\overline{W_{t}}\left(x, w, y ; \epsilon_{t}\right)=\max _{w^{\prime}}\left\{\pi_{t}\left(w-\gamma w^{\prime}+\overline{P_{t}}\left(x, y ; \epsilon_{t}\right)\right)+V_{t+1}\left(y-D_{t}\left(\epsilon_{t}\right), w^{\prime}\right)\right\}
$$

with the boundary condition $V_{T}(x, w)=\pi_{T}\left(w+\overline{P_{T}}\left(x, y ; \epsilon_{t}\right)\right), V_{T+1}(x, 0)=0$.

According to [17], we adopt the "general certainty equivalent" operator $\phi(\cdot)$ defined on a function $g(\cdot)$ of an ambiguous uncertainty $\xi$, i.e, $\Phi_{\Omega}^{R}[\phi(\xi)]=\min _{g_{\epsilon} \in \Omega}-R \ln E_{g_{\epsilon}}\left[\mathrm{e}^{-\frac{\phi(\xi)}{R}}\right]$. Note that $\Phi_{\Omega}^{R}=C E_{\xi}^{R}$ when $\Omega$ is a singleton. Obviously, the operator $\Phi_{\Omega}^{R}$ generalizes the certainty equivalent operator $C E_{\xi}^{R}$ in Section 4 .

Assume that $\pi_{t}(f)=-\alpha_{t} \mathrm{e}^{-\frac{f}{\beta_{t}}}$, and the ambiguity sets satisfy certain technical conditions so that the minimization in the general certainty equivalent operator can always be attained. Similar to the proof of Lemma 3, the stochastic cash balance problem in the ambiguity and risk averse model (16) (17) can be calculated through the following dynamic programming

$$
\begin{aligned}
G_{t}(x)= & \max _{y}\left\{-K \delta(y-x)-Q \delta(x-y)-k(y-x)^{+}-q(y-x)^{-}\right. \\
& \left.+\Phi_{\Omega_{t}}^{R_{t}}\left[P_{t}\left(y ; \epsilon_{t}\right)+\gamma G_{t+1}\left(y-D_{t}\left(\epsilon_{t}\right)\right)\right]\right\}
\end{aligned}
$$

with boundary condition $G_{T+1}(x)=0$.

To obtain the structure on the optimal policies, we need the following result, which implies the minimum envelope of $(K, Q)$-concave functions is still $(K, Q)$-concave.

Proposition 2 If $f(x, v)$ is $(K, Q)$-convex in $x$ for any $v$, then $g(x)=\max _{v} f(x, v)$ is also $(K, Q)$-convex.

Proof For any $x_{0} \leq x_{1}$ and $\lambda \in[0,1], x_{\lambda}=(1-\lambda) x_{0}+\lambda x_{1}$, we have

$$
\begin{aligned}
g\left(x_{\lambda}\right) & =\max _{v} f\left((1-\lambda) x_{0}+\lambda x_{1}, v\right) \\
& \leq \max _{v}\left[(1-\lambda) f\left(x_{0}, v\right)+\lambda f\left(x_{1}, v\right)+\lambda K+(1-\lambda) Q-\min \{\lambda, 1-\lambda\} \min \{K, Q\}\right] \\
& \leq \max _{v}\left[(1-\lambda) f\left(x_{0}, v\right)\right]+\max _{v}\left[\lambda f\left(x_{1}, v\right)\right]+\lambda K+(1-\lambda) Q-\min \{\lambda, 1-\lambda\} \min \{K, Q\} \\
& =(1-\lambda) g\left(x_{0}\right)+\lambda g\left(x_{1}\right)+\lambda K+(1-\lambda) Q-\min \{\lambda, 1-\lambda\} \min \{K, Q\} .
\end{aligned}
$$

Note that Proposition 2 also holds for $K$-concave and symmetric- $K$-concave since $K$-concave function and symmetric- $K$-concave are both special cases of $(K, Q)$-concave function.

Then, combined with Proposition 2, similar to the proof of the exponential utility function case, it is easy to see that Theorems 4 and 5 hold for the stochastic cash balance problem under the exponential utility function with ambiguity aversion.

\section{Conclusions}

In this paper, we propose a framework for incorporating risk aversion in stochastic cash balance problem. We characterize the structure of the optimal policy on the risk-averse stochastic cash balance problem according to the consumption model. We show that the structure of the optimal policy for a decision maker with exponential utility function is almost identical to the structure of the optimal risk-neutral operations policy. Furthermore, we extend the results for the exponential utility function to the ambiguity aversion case. 


\section{References}

[1] Arrow K J, Karlin S, Scarf H. Studies in the mathematical theory of inventory and production. Stanford University Press, Stanford, California, 1958.

[2] Eppen G D, Fama E F. Cash balance and simple dynamic portofolio problems with proportional costs. International Economics Review, 1969, 10: 119-133.

[3] Whisler W D. A stochastic inventory model for rented equipment. Management Science, 1967, 13: 640-647.

[4] Feinberg E L, Lewis M E. Optimality of four-threshold policies in inventory systems with customer returns and borrowing/storage options. Probability in the Enginering and Informatin Sciences, 2005, 19(1): 45-71.

[5] Girgis N M. Optimal cash balance levels. Management Science, 1968, 15(3): 130-140.

[6] Neave E H. The stochastic cash balance probem with fixed costs for increases and decreases. Management Science, 1970, 16(7): 472-490.

[7] Chen X, Simchi-Levi D. A new approach for the stochastic cash balance problem with fixed cost. Probability in the Engineering and Informational Science, 2009, 23(4): 545-562.

[8] Ye Q, Duenyas I. Optimal capacity investment decision with two-sided fixed capacity adjustment costs. Operations Research, 2007, 55(2): 272-283.

[9] Feinberg E L, Lewis M E. Optimality inequalities for average cost Markov decision processes and the stochastic cash balance problem. Mathematics of Operations Research, 2007, 32(4): 769-783.

[10] Chen Y H, Xu M H, Zhang Z G. A risk-averse newsvendor model under CVaR criterion. Operations Research, 2009, 57(4): 1040-1044.

[11] Eeckhoudt L, Gollier C, Schlesinger H. The risk-averse (and prudent) newsboy. Management Science, 1995, 41(5): 786-794.

[12] Lau H S. The newsboy problem under alternative optimization objective. Journal of the Operational Research Society, 1980, 31(6): 525-535.

[13] Wu M, Zhu S X, Teunter R H. Newsvendor problem with random shortage cost under a risk criterion. International Journal of Production and Economics, 2013, 145: 790-798.

[14] Bouakiz M, Sobel M J. Inventory control with an exponential utility criterion. Operations Research, 1992, 40(3): 603-608.

[15] Chen X, Sim M, Simchi-Levi D, et al. Risk aversion in inventory management. Operations Research, 2007, 55(5): 828-842.

[16] Nilim A, EI Ghaoui L. Robust solutions to Markov decision problems with uncertain transition matrices. Operations Research, 2005, 53(5): 780-798.

[17] Chen X, Sun P. Optimal structural policies for ambiguity and risk averse inventory and pricing models. SIAM Journal on Control and Optimization, 2012, 50(1): 133-146.

[18] Scarf H. The optimality of $(s, S)$ policies in the dynamic inventory problem. Proceedings of the 1st Stanford Symposium on Mathematical Methods in the Social Sciences, Stanford University Press, Stanford, California, 1960.

[19] Chen X, Simchi-Levi D. Coordinating inventory control and pricing strategies with random demand and fixed ordering cost: The finite horizon case. Operations Research, 2004, 52(6): 887-896. 